\title{
Electronic Devices from Conducting Organics and Polymers
}

\author{
Richard S. Potember, Robert C. Hoffman, Henry S. Hu, \\ James E. COCCHIARO, Carla A. VIANDS, \\ and Theodore O. POEHLER
}

The Johns Hopkins University, Applied Physics Laboratory, Laurel, MD 20707, U.S.A.

(Received August 29, 1986)

\begin{abstract}
In the past 20 years, organic molecular crystals and conducting organic polymers have been prepared in a variety of forms where electrical conductivity can be systematically controlled over a range of 10 orders of magnitude. Recently, a number of potential applications have emerged from this research. This paper provides a brief overview of current research into the possibility of using organic polymers and charge-transfer complexes to fabricate electronic and optical devices.

KEY WORDS Molecular Electronics / Batteries / Conductive Polymers / Molecular Crystals / Polyacetylene / Polypyrrole / Optical Recording Disk / Photochromics / Tetracyanoquinodimethane / Electrochromics /
\end{abstract}

The rapid development in the semiconductor industry has been marked by the increasing density and complexity of semiconductor chip circuitry. Considerable attention has been given to the inherent problems associated with increasing the density of integrated circuitry. In the future, fundamental physical considerations will set limits on the size and properties of integrated circuits that can be manufactured by conventional techniques. Some of the limitations arise from processing considerations, materials properties and operational constraints.

Although semiconductor technology has not yet reached its full potential, future generations of computers may require revolutionary new technologies based on totally different types of materials to increase memory and processing capabilities. One possible class of materials which may find applications in the electronics industry is carbon based (organic) semiconductors and conductors.

The replacement of inorganic semiconductors and metals by organic macromolecular, polymeric, or even biological materials has recently been termed Molecular Electronics. This new interdisciplinary field, combining organic chemistry, solid-state physics, and microelectronic engineering, offers the potential of revolutionizing electronic and computer speed and capacity by making devices from complex molecular structures.

Organic compounds and polymers offer a viable alternative to the traditional inorganics in many applications because of their extremely small size, abundance, diversity, ease of fabrication, and potential low cost. For example, the typical dimensions of organic molecular structures (10-100 Angstroms) are two to three orders of magnitude smaller than existing and proposed devices developed by current state-of-the-art lithographic techniques. Organic semiconductors offer an additional feature in that it is possible to control the electronic and optical properties of an organic device by altering or tailoring the organic molecular structure before fabricating the device. In molecular electronic devices 


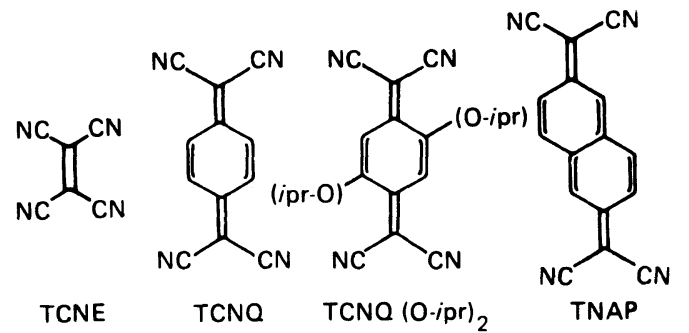

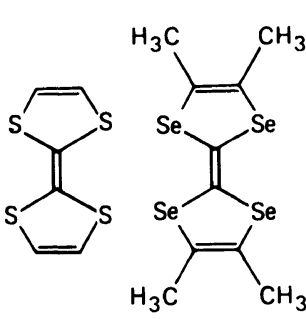

TTF<smiles>C1CSC2=C(S1)SC(=C1SC3=C(SCCS3)S1)S2</smiles>

BEDT<smiles>N#CC(C#N)=c1c(F)c(F)c(=C(C#N)C#N)c(F)c1F</smiles>

TCNO F 4
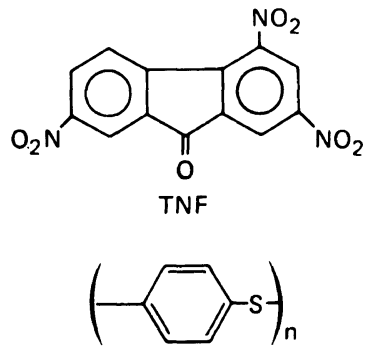

Poly(p-phenylene sulfide) (PPS)

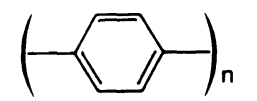

Poly (p-phenylene) (PPP)<smiles>C=Cn1c2ccccc2c2ccccc21</smiles>

$\mathrm{N}$ - vinylcarbazole
Figure 1. Structural formulas of organic donor and acceptor molecules.

the electronic and/or optical properties are locked into the molecular structure instead of produced by the processing technique. This feature called molecular architecture, or molecular engineering, may greatly simplify future manufacturing processes by reducing the number of fabrication steps, hence increasing density, speed, and production yield.

Current research in molecular electronics centers on the design and fabrication of new synthetic molecular materials, evaluating these materials, and applying them to novel technical applications. Research in this field is cur- rently divided into two areas: (1) electro-active polymers sometimes called plastic metals or synmetals (synthetic metals); (2) molecular crystals which include monomeric compounds and organic charge-transfer complexes. Figure 1 shows the structural formula of several common electroactive polymers and monomeric compounds discussed in this paper.

\section{CONDUCTING ORGANIC POLYMERS: AN OVERVIEW}

The study of electronic transport in organic polymers is more than thirty years old. The first organic polymers prepared were electrically insulating with conductivities as low as $10^{-14}$ (ohm-centimeters) $^{-1}$. The insulating properties are the result of all of the electrons in the polymer being localized in the hybridatom molecular orbital bonds i.e., the saturated carbon framework of the polymer. These insulators, which include polymers such as $\operatorname{poly}(n$-vinylcarbozole), or polyethylene, have extremely large band gaps with energy as high as 10 electron volts required to excite electrons from the valence to the conduction band. Electrical applications of insulating organic polymers are limited to insulating or supporting materials where low weight and excellent processing and mechanical properties are desirable.

Semiconducting organic polymers, sometimes called electroactive polymers, exhibit conductivities from $10^{-9}$ to 100 (ohmcentimeters $)^{-1}$. These polymers can be classified into three groups based on their chemical structure: (1) composite polymers which combine graphite-like structures with metal particles. The concentration of metal particles may often reach $85 \%$ by weight in the composite polymers; (2) polymeric charge-transfer complexes in which a cationic species, often a metal ion or an anionic species such as the iodide ion $\left(\mathrm{I}_{3}\right)^{-}$or an organic acceptor such as TCNQ or TNF (trinitrofluorenone) interacts with the polymer main chain by altering the 
Electronic Devices from Conducting Organics and Polymers

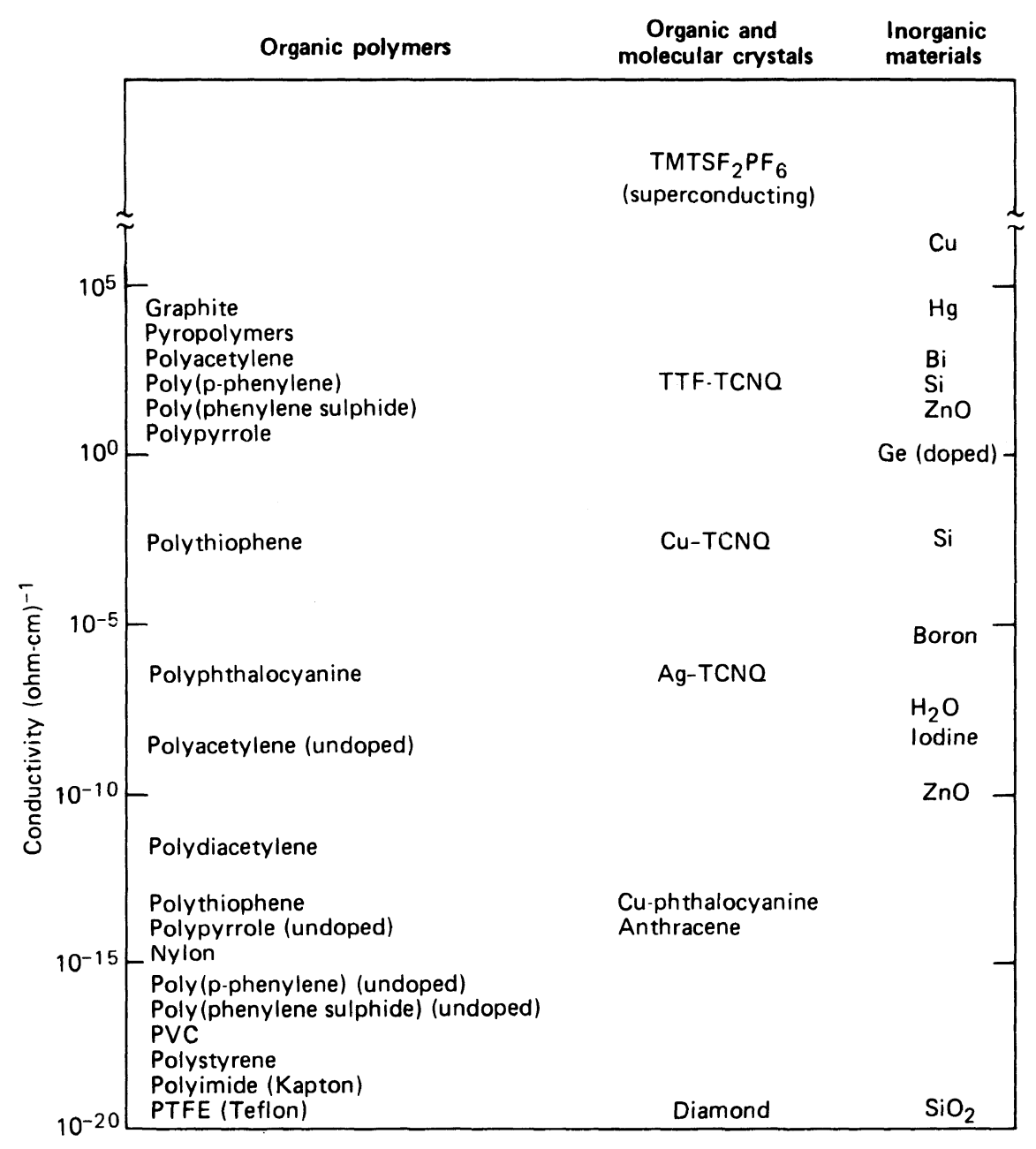

Figure 2. Compilation of electrical conductivities of organic polymers.

molecular orbital arrangement through electron interactions, and; (3) conjugated polymers which show semiconducting properties because the $\pi$-orbital overlap along the unsaturated polymer chain allows conduction electron to interact with neighboring $\pi$-orbitals on adjacent carbon atoms.

High electrical conductivity has been observed in several conjugated polymer or polyene systems (Figure 2). The first and simplest organic polymer to show high conductivity was "doped" polyacetylene (Figure 3 ). In the "doped" form its conductivity is in excess of 200 (ohm-centimeters) ${ }^{-1}$. Although polyacet- ylene was first prepared some time ago, it was not until 1977 that this polyene was modified by combining the carbon chain with iodine and other molecular acceptors to produce a material with metallic conductivity. ${ }^{2}$

The actual nature of the electrical conductivity in doped and undoped polyacetylene is still highly speculative. However, several possible explanations have been developed to explain the properties of these polymers. In an ideal infinite chain length of polyacetylene, the pi-electrons would be expected to form a halffilled energy band leading to one-dimensional metallic behavior. In reality, however, a finite 


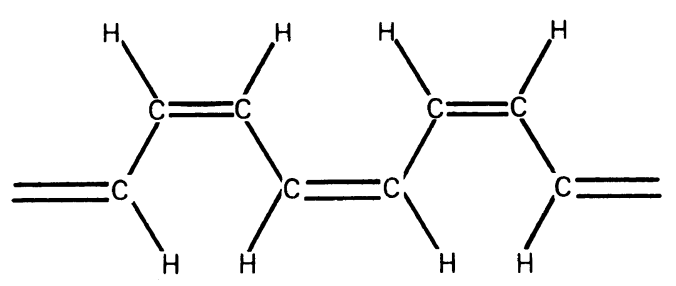

(a)

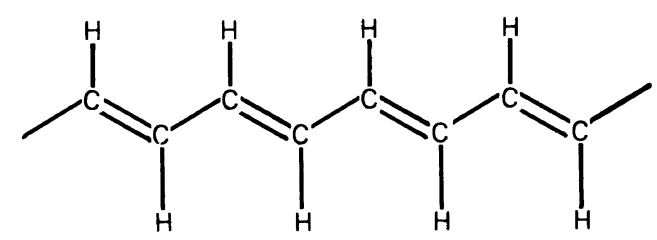

(b)

Figure 3. (a) cis-Forms in idealized polyacetylene chain. (b) trans-Forms in idealized polyacetylene chain.

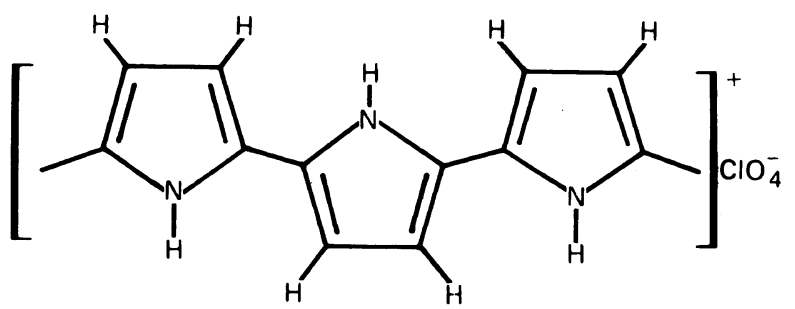

Figure 4. Chemical structure of conductive polypyrrole.

length of $(\mathrm{CH})_{x}$ chains would be an intrinsic semiconductor with an electron band gap of approximately 2 electron volts because the alternating double bond-single bond structure has two atoms per unit cell instead of one; hence a semiconductor band gap is formed through a Peierls metal-to-semiconductor transition.

In the ten years since the discovery of the first highly conductive organic polymer, work on numerous other conductive polyene systems (Figure 1) including poly ( $p$-phenylene) PPP, $\operatorname{poly}(p$-phenylene sulfide) PPS, polypyrrole PP, and poly ( $p$-phenylene vinylene) PPV has proved that, as a class of materials, the unsaturated conjugated organic polymers can be in an electrically conducting form and still maintain the low density, processibility and elasticity that make polymers unique materials.

One of the most scientifically and technologically studied new conductive organic polymers is polypyrrole (Figure 4). The highly stable, flexible films of polypyrrole produced by the one-step electro-oxidation have room temperature $p$-type conductivities ranging from 10 to 100 (ohm-centimeters) $^{-1}$. No additional dopants are required to produce electrical conductivity. ${ }^{3}$

In addition to showing high electrical conductivity, polypyrrole films can be repeatedly electrochemically driven or "switched" between a black highly conductive 100 (ohm-

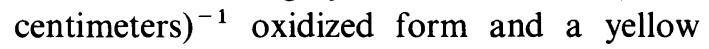
nonconducting neutral. The switching rate for thin films is approximately one per second. ${ }^{4}$ 


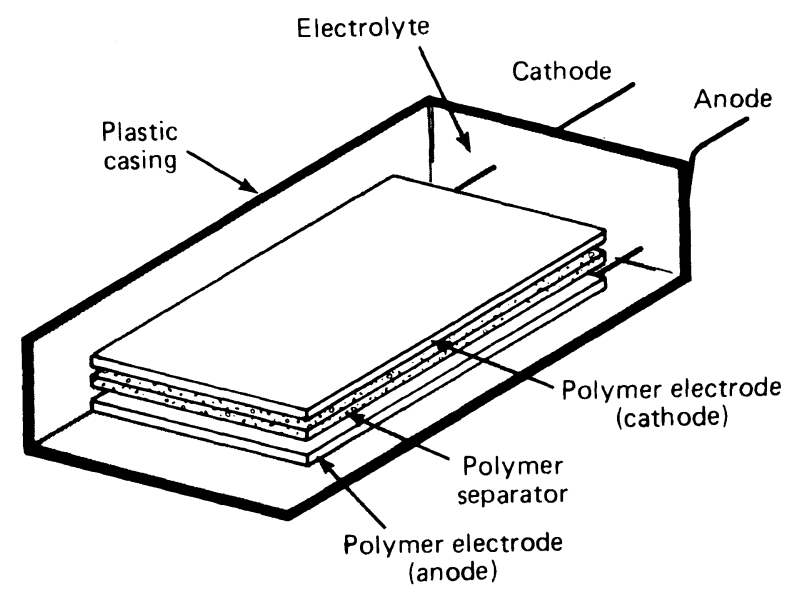

Figure 5. Schematic of "plastic" battery.

\section{APPLICATIONS OF MOLECULAR ELECTRONIC MATERIALS}

Molecular electronic materials show great promise in a wide range of applications. Many of the uses will be in direct competition with existing inorganic materials, while the novel properties reported in many molecular crystals and polymers assure that entirely new applications and technologies will emerge from this field.

A partial list of applications currently under development includes capacitors, transistors, batteries, memory devices, chemical sensors, transducers, and plastic wiring. A complete description of each application and material is lengthy; however, five novel areas of application are described briefly below to illustrate the role these materials may play in future technologies.

\section{LIGHTWEIGHT RECHARGEABLE BATTERIES}

Highly conductive organic polymers are being evaluated for electrode materials in both disposable and rechargeable batteries. When two sheets of polyacetylene or $\operatorname{poly}(p$ phenylene) are separated by an insulating film of polycarbonate saturated in an electro- lyte (lithium perchlorate), and completely encapsulated in a plastic casing a "plastic battery" can be made 5 (Figure 5). The two sheets of polyacetylene or poly ( $p$-phenylene) act as both anode and cathode for the battery.

These novel polymeric batteries have several notable features. They are rechargeable, ten times lighter than a conventional lead-acid battery, and flexible enough to fit into a variety of design configurations.

\section{PHOTOVOLTAIC APPLICATIONS}

Several conductive polymers are being tested for use as coatings on photovoltaic and photoelectroactive semiconductors in solar energy conversion. As an example, a film of conductive polypyrrole used as an overcoat on the activated semiconductor, cadmium sulfide, can enhance the evolution of oxygen at the electrode/electrolyte surface and increase the lifetime of the cell by a million fold. ${ }^{6}$

In another example, a photovoltaic cell has been fabricated using polyacetylene as the actual active photoelectrode. In a Schottky barrier configuration, this solar cell delivered an open circuit voltage of 0.3 volts and a short circuit current of 40 microamperes per square centimeter under an illumination equivalent to one sun. ${ }^{7}$ Polyacetylene may be an attrac- 
(a)

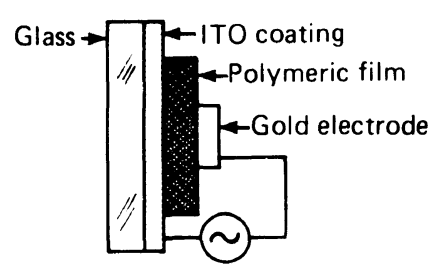

(b)

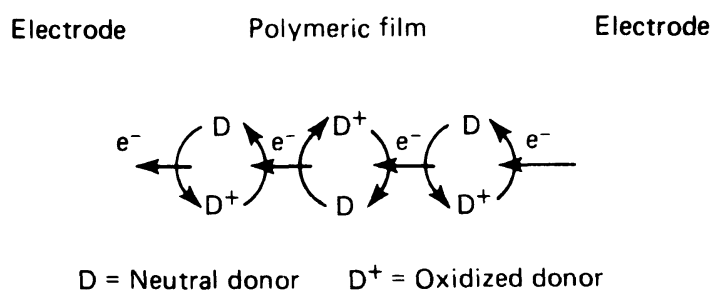

Figure 6. (a) Cell structure of electrochromic display. (b) Coloring electrochromic mechanism in polymeric film.

tive solar cell material because studies suggest it is a direct band gap semiconductor with an optical band gap matching the solar spectrum. The electronic structure is analogous to traditional inorganic semiconductors in that the strong $\pi$-electron overlap along the polymer chain leads to large energy band widths.

\section{ELECTROCHROMIC POLYMER DISPLAYS}

An electrochromic display is a thin solid state device that changes color reversibly when subjected to a small electrical potential. This new display technology has several advantages over liquid crystal displays currently in use. Electrochromic displays have low power consumption, good optical contrast, a wide viewing angle, and an all solid state construction.

In a unique application of charge-transfer organic materials, electrochromism has been reported in a thin film system consisting of a polymer, polymethacrylonitrile (PMCN), and organic redox materials such as TTF or pyrazoline. $^{8}$ An electrochromic display cell structure using these materials is shown in Figure 6a. It consists of a transparent glass electrode, the electrochromic polymer system and a thin gold semitransparent counterelectrode.

When the structure is electrically biased (1.0-3.5 volts), the optical density of the film changes, i.e., the color changes, because the organic complex undergoes a reversible electron transfer reaction from a neutral form to an oxidized form (Figure 6b). Using a TTF redox compound, the color of the display changes from yellow to a bright red, while pyrazoline switches from transparent to yellow. Multicolor displays are also being studied which use combinations of organic compounds that exhibit multiple coloration at different applied potentials.

\section{AN ERASABLE COMPACT DISK}

The interaction of laser radiation with matter for use in optical storage systems has received considerable attention. A variety of different media, including photographic films, photoresists, photosensitive polymers, photochromics, thin amorphous films, and electrooptic materials have been proposed as opti- 
cal storage systems. Present optical storage systems have been applied in the fields of document storage, audio and video reproduction, and direct data collection. However, optical storage systems have not seen wide-spread applications in computer technology because most optical media are not erasable.

We have recently developed an erasable optical recording medium using the electric field induced optical switching effect in films of either copper or silver complexed with the electron acceptors tetracyanoethylene (TCNE), tetracyanonaphthoquinodimethane (TNAP), tetracyanoquinodimethane (TCNQ), or other derivatives of TCNQ (Figure 1)..$^{-15}$ The optical change in these materials is reversible and fast, with switching times of less than 10 nanoseconds observed in static switching experiments.

The observed changes in the optical properties of films fabricated from these organic materials is a direct consequence of the laser induced reaction shown in eq 1 .

$$
\begin{aligned}
& n\left[\mathrm{M}^{+}\left(\mathrm{TCNQ}^{-}\right)\right] \underset{\text { heat }}{\stackrel{\boldsymbol{E}}{\rightleftarrows}} x \mathrm{M}^{\circ} \\
& \quad+n-x\left[\mathrm{M}^{+}\left(\mathrm{TCNQ}^{-}\right)\right]+x\left(\mathrm{TCNQ}^{\circ}\right)
\end{aligned}
$$

where $\mathbf{M}$ can be silver, copper, sodium, potassium, or lithium.

The reaction shown in eq 1 is reversible and the thermodynamically favored initial phase can be readily reformed by heating the com- plex phase (right side of equation) of the salt. Various diagnostic techniques including infrared, Auger, X-ray photoelectron, and Raman spectroscopy have been applied to help understand this reaction. These experiments suggest that the effect of the applied electric field (laser) on an organometallic chargetransfer salt (e.g., copper TCNQ) is to induce a phase transition to a non-stoichiometric complex salt containing neutral acceptor molecules $\left(\mathrm{TCNQ}^{\circ}\right)$.

Figure 7 shows a cross section of a typical erasable optical disk medium using the TCNQ family of organometallic materials. An approximately 2000 Angstrom-thick organic charge-transfer salt is deposited on aluminum, glass or polycarbonate supporting base using a solid-state diffusion process. This solid-state diffusion/deposition guarantees that the organic film will be uniform over a wide area and that the size of the individual crystallites (less than 1 micron) will be minimized. The disk is completed when a thin protective polymer overcoat is cast over the photoactive organic layer.

The disk can then be placed in an optical recording, playback and erasure system. A block diagram of a typical laser diode optical recording system is shown in Figure 8. Information is recorded, read and erased on the spinning disk by modulating the intensity of the laser. To record information, the in-

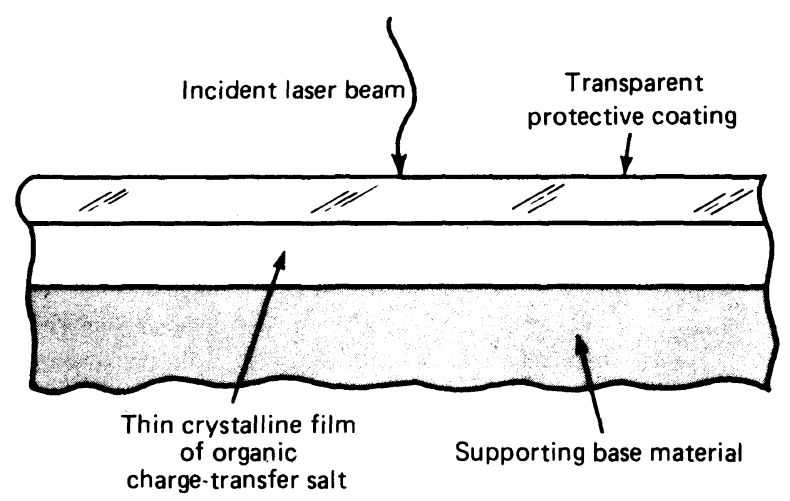

Figure 7. Schematic of optical storage disk using organic charge-transfer complexes. 


\section{R. S. Potember et al.}

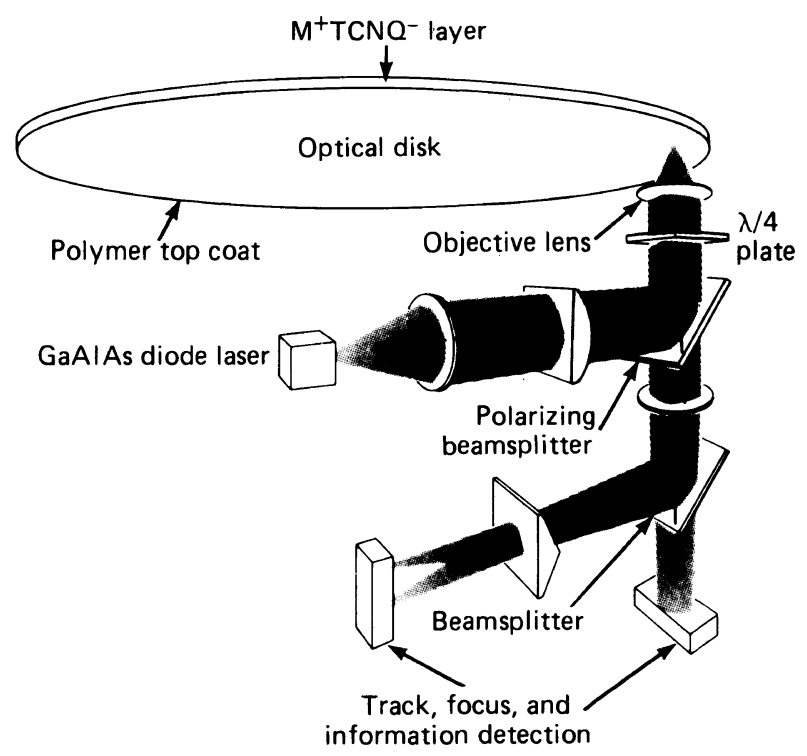

Figure 8. Block diagram of typical optical recording system.

tensity of the modulated laser is increased above the power threshold of the particular organic charge-transfer complex, thus producing a series of high contrast spots on the surface of the rotating recording medium. The reading of stored information is accomplished by illuminating each bit location with a continuous reduced power beam (below write threshold) and monitoring the reflected intensity with a suitable photodetector which converts the light into electrical impulses. The information can subsequently be erased by adjusting the power level and pulse duration to cause localized heating on the media.

An example of optical information storage using this organic medium is shown in Figure 9. The figure shows the magnified surface of a AgTCNQ optical disk. Note the high contrast between the optically switched regions (spots) and the background. The spots were made with the 532 nanometer line from a pulsed $\mathrm{Nd}$ :YAG laser; laser energy was about 5 microjoules per pulse. Spot size was approximately 20 microns.

We have irradiated a number of other copper and silver TCNQ type charge-transfer

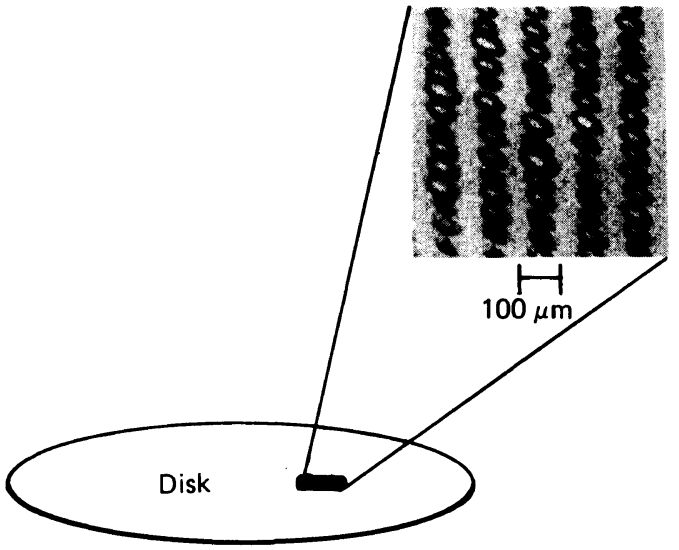

Figure 9. Magnified surface of AgTCNQ optical disk showing high contrast between optically switched and unswitched regions for various laser intensities.

complexes with the 458,488 and 514-nanometer lines from an argon ion laser, the 532 and 1064-nanometer lines from a Nd:YAG laser, the 633 nanometer line from a $\mathrm{He}: \mathrm{Ne}$ laser, the 780 nanometer line from a GaAlAs laser and the 10600 nanometer line from a carbon dioxide laser. In the majority of materials studied, the write threshold power is relatively independent of wavelength throug- 
hout the visible and infrared region of the spectrum. The threshold writing power varies between 3 and 150 milliwatts depending upon the specific choice of metal and acceptor complex. These power levels are well within the capabilities of most moderately powered commercial laser systems.

\section{PHOTOCHROMIC PROPERTIES OF METAL-ORGANIC CHARGE- TRANSFER COMPLEXES}

An area of technological interest is the change in the macroscopic optical properties of these organometallic materials in an electromagnetic field. The potential for large changes in transmission and reflection in CuTCNQ and other members of this family make these compounds prime candidates as high speed photochromic filters. ${ }^{14,16}$ We have observed that these films undergo a change from their respective blue and violet colors to a rather pale yellow color characteristic of neutral TCNQ. As shown in Figure 10a, a typical CuTCNQ film formed by solid state diffusion is rather poorly transmitting from the midvisible into the near infrared in the vicinity of 1100 nanometers, and there is a substantial increase in transmission extending into the infrared. Upon irradiating the CuTCNQ film with a Nd:YAG laser at 532 nanometers, one observes a significant increase in transmission throughout the spectrum ranging from the midvisible to the infrared. Dramatic increases are observed in the red end of the spectrum and in the near infrared, those regions which are of particular interest with respect to many of the principal laser sources.

The transmission properties of an AgTCNQ film are shown in Figure 10b. In contrast to the changes observed in CuTCNQ, the switched and unswitched films are quite similar in the infrared portion of the spectrum. Large changes in transmission are noted in the visible part of the spectrum between 400 and 600 nanometers. The switched AgTCNQ be-

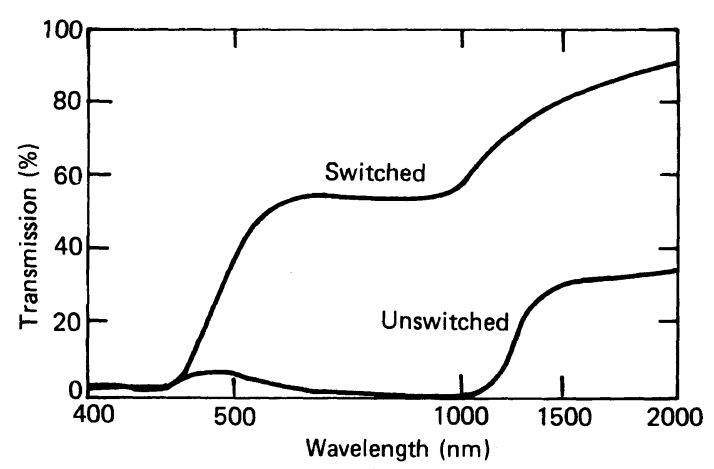

Figure 10a. Typical CuTCNQ transmission switch30 millijoule pulses from frequency doubled Nd:YAG laser (532 nanometers).

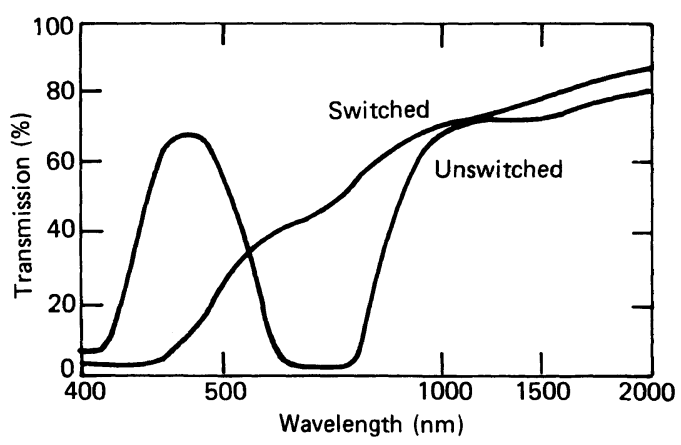

Figure 10b. Typical AgTCNQ transmission switch45 millijoule pulses from frequency doubled $\mathrm{Nd}$ : YAG laser (532 nanometers).

comes strongly transmitting in a band centered at 500 nanometers, while the unswitched film is poorly transmitting in the same region.

\section{CONCLUDING REMARKS}

It may be premature to evaluate the technological significance of this new field of science to the electronics industry. However, this paper provides concrete examples of organic macromolecules and polymers with specific chemical and physical properties useful in device applications such as lightweight rechargeable batteries, photovoltaic solar cells, junction and electro-optic devices, photochromic filters, and information storage and processing media. 
In each of the various devices and applications discussed in this paper, the novel concept of molecular architecture is demonstrated i.e., the precise design and synthesis of chemical structure controls the specific physical properties observed in a particular device application. Current research in this field has also established a foundation of materials, and fundamental scientific principles amenable to the design of new molecular electronic devices.

Acknowledgments. Work at the Applied Physics Laboratory was supported in part by DARPA/AFOSR, Wright-Patterson Air Force Base, and U. S. Naval Air Systems Command under contract No. N00024-85-C5301.

\section{REFERENCES}

1. Y. W. Park, A. J. Heeger, M. A. Druy, and A. G. MacDiarmid, J. Chem. Phys., 73, 946 (1980).

2. C. K. Chiang, C. R. Fincher, Jr., Y. W. Park, A. J. Heeger, H. Shirakawa, E. J. Louis, S. C. Gau, and A. G. MacDiarmid, Phys. Rev. Lett., 39, 1098 (1977).

3. K. K. Kanazawa, A. F. Diaz, W. D. Gill, P. M. Grant, G. B. Street, G. P. Gardini, and J. F. Kwak, Synthetic Metals, 1, 329 (1980).

4. P. G. Pickup and R. A. Osteryoung, J. Am. Chem.
Soc., 106, 2294 (1984).

5. P. J. Nigrey, D. MacInnes, D. P. Nairns, and A. G. MacDiarmid, J. Electrochem. Soc., 128, 1651 (1981); R. Somano, Appl. Phys. Commun., 1, 179 (1981-82).

6. R. A. Bull, F. R. Fan, and A. J. Bard, J. Electrochem. Soc., 130, 1636 (1983); A. J. Frank and K. J. Honda, J. Phys. Chem., 86, 1933 (1982).

7. S. N. Chen, A. J. Heeger, Z. Kiss, A. G. MacDiarmid, S. C. Gau, and D. L. Peebles, Appl. Phys. Lett., 36, 96 (1980).

8. Y. Hirai and C. Tani, Appl. Phys. Lett., 43, 704 (1983).

9. R. S. Potember, T. O. Poehler, and D. O. Cowan, Appl. Phys. Lett., 34, 405 (1979).

10. R. S. Potember, T. O. Poehler, A. Rappa, D. O. Cowan, and A. N. Bloch, J. Am. Chem. Soc., 102, 3659 (1980).

11. R. S. Potember, T. O. Poehler, D. O. Cowan, A. N. Bloch, P. Brant, and F. L. Carter, Chem. Scripta, 17, 219 (1981).

12. R. S. Potember, T. O. Poehler, D. O. Cowan, and A. N. Bloch, Proceedings of the NATO Conference on Chemistry and Physics of One-Dimensional Materials, L. Alcacer, Ed., Reidel, Boston, 1980, p 419.

13. R. S. Potember, T. O. Poehler, and R. C. Benson, Appl. Phys. Lett., 41, 548 (1982).

14. R. S. Potember, R. C. Hoffman, R. C. Benson, and T. O. Poehler, J. De Physique, 44, C3-1597 (1983).

15. R. C. Benson, R. C. Hoffman, R. S. Potember, E. Bourkoff, and T. O. Poehler, Appl. Phys. Lett., 42, 855 (1983).

16. T. Hirono, M. Fukuma, and T. Yamada, J. Appl. Phys., 57, 2267 (1985). 